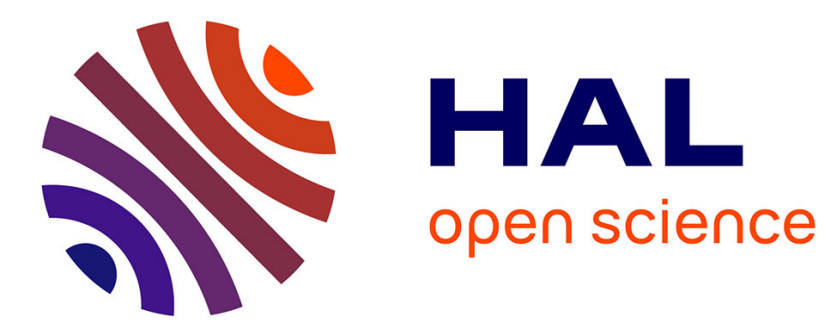

\title{
Dynamic Parameter Identification of Actuation Redundant Parallel Robots: Application to the DualV
}

Sébastien Briot, Maxime Gautier, Sébastien Krut

\section{To cite this version:}

Sébastien Briot, Maxime Gautier, Sébastien Krut. Dynamic Parameter Identification of Actuation Redundant Parallel Robots: Application to the DualV. AIM: Advanced Intelligent Mechatronics, Jul 2013, Wollongong, Australia. pp.637-643, 10.1109/AIM.2013.6584164 . hal-00818104

\section{HAL Id: hal-00818104 https://hal.science/hal-00818104}

Submitted on 11 May 2016

HAL is a multi-disciplinary open access archive for the deposit and dissemination of scientific research documents, whether they are published or not. The documents may come from teaching and research institutions in France or abroad, or from public or private research centers.
L'archive ouverte pluridisciplinaire HAL, est destinée au dépôt et à la diffusion de documents scientifiques de niveau recherche, publiés ou non, émanant des établissements d'enseignement et de recherche français ou étrangers, des laboratoires publics ou privés. 


\title{
Dynamic Parameter Identification of Actuation Redundant Parallel Robots: Application to the DualV
}

\author{
Sébastien Briot ${ }^{1}$, Maxime Gautier ${ }^{2}$ and Sébastien Krut $^{3}$
}

\begin{abstract}
Off-line robot dynamic identification methods are based on the use of the Inverse Dynamic Identification Model (IDIM), which calculates the joint forces/torques (estimated as the product of the known control signal - the input reference of the motor current loop - by the joint drive gains) that are linear in relation to the dynamic parameters, and on the use of linear least squares technique to calculate the parameters (IDIM-LS technique). However, as actuation redundant parallel robots are over-constrained, their IDIM has infinity of solutions for the force/torque prediction, depending on the value of the desired overconstraint that is a priori unknown in the identification process. As a result, the IDIM cannot be used as it.

This paper proposes a modified formulation for the IDIM of actuation redundant robots that can be used for identification purpose. This formulation consists of projecting the input torques/forces on the platform coordinates, thus leading to a unique solution of the model that can thus be used in the identification process. The identification of the inertial parameters of a planar parallel robot with actuation redundancy, the DualV, is then carried out using this modified IDIM. Experimental results show the validity of the method.
\end{abstract}

\section{INTRODUCTION}

Parallel robots are increasingly being used since a few decades. This is due to their main advantages compared to their serial counterparts that are: (i) a higher intrinsic rigidity, (ii) a larger payload-to-weight ratio and (iii) higher velocity and acceleration capacities [1]. However, their main drawback is probably the presence of singularities in the workspace. In order to overcome this difficulty, actuation redundancy can be used [2], [3]. Actuation redundancy means that the robot has more actuators than degrees of freedom (dof) to control and is thus over-constrained. Overconstraints can be smartly used to improve the robot properties, such as increasing the acceleration or payload capacities [4] or even decreasing the backlash [5]. However, this involves the use of more complicated controllers.

Several control approaches could be envisaged [6], [7], but it appears that, for high-speed robots or when varying loads have to be compensated (e.g. in pick-and-place operations or machining), computed torque control is generally used [5], [8]. This approach requires an accurate identification of the

This work was supported by the French ANR ARROW (ANR 2011BS3 $00601)$

${ }^{1} \mathrm{~S}$. Briot is with the French CNRS and the Institut de Recherche en Communications et Cybernétique de Nantes (IRCCyN), 44321 Nantes France (Sebastien.Brioteirccyn.ec-nantes.fr)

${ }^{2} \mathrm{M}$. Gautier is with the LUNAM, Universite de Nantes and the IRCCyN, 44321 Nantes France (Maxime.Gautierdirccyn.ec-nantes.fr)

${ }^{3} \mathrm{~S}$. Krut is with the French CNRS and the Laboratoire d'Informatique, de Robotique et de Microélectronique de Montpellier (LIRMM), 34095 Montpellier France (Sebastien.Krutelirmm.fr) dynamic model of the robot with the load [9], which can be obtained if two main conditions are satisfied:

1) a well-tuned derivative band-pass filtering of actuated joints position is used to calculate the actuated joints velocities and accelerations, and

2) the values of actuator drive gains $g_{\tau}$ are accurately known to calculate the actuator force/torque as the product of the known control signal computed by the numerical controller of the robot (the current references) by the drive gains

The usual identification procedure of the robot dynamic parameters requires the computation of the inverse dynamic identification model (IDIM) of the studied robot that gives the values of the input forces/torques as a function of the robot configuration, velocity and acceleration [10]. However, for actuation redundant parallel robots, the inverse dynamic model is not unique and depends on the overconstraint in the mechanism that cannot be a priori known in the identification process. Thus, identification using the usual IDIM for redundant parallel robots cannot be carried out.

In this paper, a modified formulation of the IDIM for actuation redundant parallel robots is proposed that does not require the knowledge of the overconstraint and the identification of a 3 dof planar parallel robot with actuation redundancy named the DualV [11] is carried out. To the best of our knowledge, the identification of the inertial parameters of actuation redundant parallel robots was never achieved before.

This paper is divided as follows. Section II presents some recalls on the computation of the IDIM of parallel robots without actuation redundancy. These recalls are necessary as the computation of the IDIM of actuation redundant parallel robots is partially based on some mathematical derivations shown in this part and to understand why the usual IDIM cannot be used in the identification procedure. Then, Section III presents the new formulation of the IDIM for the identification of parallel robots with actuation redundancy. Section IV briefly recalls the identification procedure and experimental results on the DualV are presented in Section V. Finally, in Section VI, conclusions are drawn.

\section{INVERSE DYNAMIC IDENTIFICATION MODEL OF PARALlEL RoBots With No ACTUATION REDUNDANCY}

\section{A. Computation of the IDIM for Parallel Robots with no Actuation Redundancy}

A parallel robot is a complex multi-body system having several closed loops (Fig. 1(a)). It is composed of a moving 


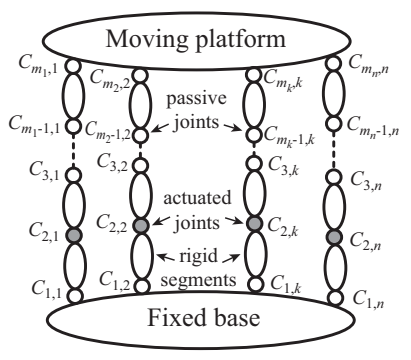

(a) Kinematic chain

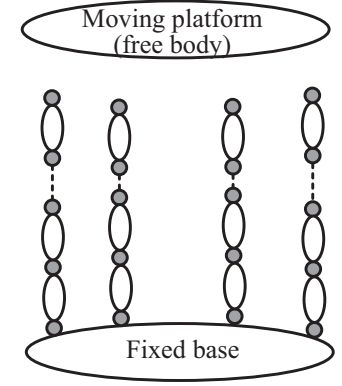

(b) Virtual tree structure
Fig. 1. A general parallel robot.

platform connected to a fixed base by $n$ legs, each composed of $m_{i}$ elements. It is considered here that there is one actuator by leg, but the method can be easily extended to robots with several actuators by legs.

The computation of IDIM of parallel robots with no actuation redundancy is decomposed into two steps [12]:

1) all closed loops are virtually opened to make the platform virtually disassembled from the rest of the structure (Fig. 1(b)); each leg joint is virtually considered actuated (even for unactuated actual joints) so that the robot becomes a tree structure with a free body: the platform; the dynamic model of the tree structure and of the free platform is then computed using a systematic procedure based on the NewtonEuler principle, and

2) the loops are then closed using the loop-closure equations and the Lagrange multipliers, which involve the computation of robot Jacobian matrices.

In what follows, the computation of the IDIM of the virtual tree structure and of the platform is recalled, and then a straightforward way to compute the Jacobian matrices for closing the loops is detailed.

\section{B. IDIM of Tree Open Loop Robots}

It is known that the complete rigid dynamic model of any open-loop tree structure can be linearly written in term of a $\left(n_{t} \times 1\right)$ vector with respect to the standard parameters $\chi_{s t_{t}}$ [10] $\left(n_{t}\right.$ denotes the total number of joints for the virtual tree structure),

$$
\tau_{i d m_{t}}\left(\mathbf{q}_{t}, \dot{\mathbf{q}}_{t}, \ddot{\mathbf{q}}_{t}\right)=\operatorname{IDM}_{s t_{t}}\left(\mathbf{q}_{t}, \dot{\mathbf{q}}_{t}, \ddot{\mathbf{q}}_{t}\right) \chi_{s t_{t}}
$$

where $\tau_{i d m_{t}}$ is the $\left(n_{t} \times 1\right)$ vector of the virtual input efforts of the tree structure, $\mathbf{I D M}_{s t_{t}}$ is the $\left(n_{t} \times n_{s t_{t}}\right)$ Jacobian matrix of $\tau_{i d m_{t}}$, with respect to the $\left(n_{s t_{t}} \times 1\right)$ vector $\chi_{s t_{t}}$ of the standard parameters given by $\chi_{s t_{t}}^{T}=\left[\chi_{s t}^{1 T}, \chi_{s t}^{2 T}\right.$, $\left.\ldots \chi_{s t}^{n_{t} T}\right]$ and $\mathbf{q}_{t}, \dot{\mathbf{q}}_{t}, \ddot{\mathbf{q}}_{t}$ are the vectors of the joint positions, velocities and accelerations, respectively.

For rigid robots, the vector $\chi_{s t}^{j}$ of link $j$ is composed of 14 standard parameters described as:

- $x x_{j}, x y_{j}, x z_{j}, y y_{j}, y z_{j}, z z_{j}$ are the 6 components of the inertia matrix of link $j$ at the origin of frame $j$,

- $m x_{j}, m y_{j}, m z_{j}$ are the 3 components of the first moment of link $j$,
- $m_{j}$ is its mass,

- $i a_{j}$ is the total inertia moment for rotor and gears,

- $f v_{j}, f s_{j}$ are the viscous and Coulomb friction coefficients in the joint, respectively, and $\tau_{o f f_{j}}=\tau_{o f f f s_{j}}+$ $\tau_{o f f \tau_{j}}$ is an offset parameter which regroups the current amplifier offset $\tau_{o f f \tau_{j}}$ and the asymmetrical Coulomb friction coefficient $\tau_{\text {offfs }}$.

In the same vein, the IDIM of the platform can be obtained as:

$$
\tau_{p}(\mathbf{x}, \mathbf{t}, \dot{\mathbf{t}})=\operatorname{IDM}_{p}(\mathbf{x}, \mathbf{t}, \dot{\mathbf{t}}) \chi_{p}
$$

where $\tau_{p}$ is the $(6 \times 1)$ vector of platform reaction wrench, $\mathbf{I D M}_{p}$ is the $(6 \times 10)$ Jacobian matrix of $\tau_{p}$, with respect to the $(10 \times 1)$ vector $\chi_{p}$ of the platform standard parameters and $\mathbf{x}, \mathbf{t}, \dot{\mathbf{t}}$ are the platform position, twist and acceleration screw, respectively.

Several methods can be used to systematically derive these equations. Here, an algorithm based on the use of the modified Denavit-Hartenberg robot geometric description and the Newton-Euler principle is applied. This modeling is known to give the dynamic model equations in the most compact form [10].

\section{IDIM of Parallel Robots with no Actuation Redundancy}

The IDIM of the virtual tree structure and of the free moving platform does not take into account the closed loop characteristics of parallel robots: among all joint and platform coordinates $\mathbf{q}_{t}$ and $\mathbf{x}$, resp., only a subset denoted as $\mathbf{q}$ is independent (the actual actuated joints positions). As a result, vectors $\mathbf{q}_{t}$ and $\mathbf{x}$ can be computed as functions of $\mathbf{q}$ using the loop-closure equations [1],

$$
\mathbf{f}_{t}\left(\mathbf{q}, \mathbf{q}_{t}\right)=\mathbf{0}, \mathbf{f}_{p}(\mathbf{q}, \mathbf{x})=\mathbf{0}
$$

Differentiating (3) with respect to time, all joint and platform velocities and accelerations can be computed using the following expressions:

$$
\begin{gathered}
\mathbf{A}_{t} \dot{\mathbf{q}}_{t}+\mathbf{B}_{t} \dot{\mathbf{q}}=\mathbf{0} \Rightarrow \dot{\mathbf{q}}_{t}=-\mathbf{A}_{t}^{-1} \mathbf{B}_{t} \dot{\mathbf{q}}=\mathbf{J}_{t} \dot{\mathbf{q}}, \\
\mathbf{A}_{p} \mathbf{v}+\mathbf{B}_{p} \dot{\mathbf{q}}=\mathbf{0} \Rightarrow \mathbf{v}=-\mathbf{A}_{p}^{-1} \mathbf{B}_{p} \dot{\mathbf{q}}=\mathbf{J}_{p} \dot{\mathbf{q}} \\
\mathbf{A}_{t} \ddot{\mathbf{q}}_{t}+\dot{\mathbf{A}}_{t} \dot{\mathbf{q}}_{t}+\mathbf{B}_{t} \ddot{\mathbf{q}}+\dot{\mathbf{B}}_{t} \dot{\mathbf{q}}=\mathbf{0} \\
\Rightarrow \ddot{\mathbf{q}}_{t}=-\mathbf{A}_{t}^{-1}\left(\dot{\mathbf{A}}_{t} \dot{\mathbf{q}}_{t}+\mathbf{B}_{t} \ddot{\mathbf{q}}+\dot{\mathbf{B}}_{t} \dot{\mathbf{q}}\right), \\
\mathbf{A}_{p} \dot{\mathbf{v}}+\dot{\mathbf{A}}_{p} \mathbf{v}+\mathbf{B}_{p} \ddot{\mathbf{q}}+\dot{\mathbf{B}}_{p} \dot{\mathbf{q}}=\mathbf{0} \\
\Rightarrow \dot{\mathbf{v}}=-\mathbf{A}_{p}^{-1}\left(\dot{\mathbf{A}}_{p} \mathbf{v}+\mathbf{B}_{p} \ddot{\mathbf{q}}+\dot{\mathbf{B}}_{p} \dot{\mathbf{q}}\right)
\end{gathered}
$$

where matrices $\mathbf{A}_{t}, \mathbf{A}_{p}\left(\mathbf{B}_{t}, \mathbf{B}_{p}\right.$, resp.) can be obtained through the differentiation of the loop-closure equations (3) with respect to all joint coordinates $\mathbf{q}_{t}$ and the platform coordinates (actuated joints positions, resp.), i.e.

$$
\begin{aligned}
& \mathbf{A}_{t}=\left[\frac{\partial \mathbf{f}_{t}}{\partial \mathbf{q}_{t}}\right], \mathbf{B}_{t}=\left[\frac{\partial \mathbf{f}_{t}}{\partial \mathbf{q}}\right] \\
& \mathbf{A}_{p}=\left[\frac{\partial \mathbf{f}_{p}}{\partial \mathbf{x}}\right], \mathbf{B}_{p}=\left[\frac{\partial \mathbf{f}_{p}}{\partial \mathbf{q}}\right]
\end{aligned}
$$

and $\mathbf{v}$ represent a subset of independent coordinates in the platform twist $\mathbf{t}(\operatorname{dim} \mathbf{v} \leq 6)$, i.e.

$$
\mathbf{t}=\mathbf{D} \mathbf{v}
$$


In the case of robots with $6 \operatorname{dof}, \mathbf{D}$ is the identity matrix.

It should be mentioned that in the case of parallel robots without actuation redundancy, the matrices $\mathbf{A}_{p}$ and $\mathbf{B}_{p}$ are square of dimension $(n \times n)$. Moreover, it is necessary to emphasize the fact that the computation of matrices $\mathbf{A}_{t}$ and $\mathbf{B}_{t}$ is generally not straightforward. Therefore, it is preferable to:

1) express the kinematic relation between the independent coordinates $\mathbf{v}_{t k}$ of the twists for all leg extremities $C_{m_{k}, k}$ and the velocities of all joints $\dot{\mathbf{q}}_{t}, \mathbf{v}_{t k}=$ $\mathbf{J}_{k} \dot{\mathbf{q}}_{t}$ (the matrix $\mathbf{J}_{k}$ stackles all Jacobian matrices corresponding to the displacements of the last joint for each serial legs and is a square matrix of dimension $\left.\left(\left(n\left(m_{i}-1\right)\right) \times\left(n\left(m_{i}-1\right)\right)\right)\right)$,

2) express the kinematic relation between the platform velocities $\mathbf{v}$ and the velocities $\mathbf{v}_{t k}$ of all leg extremities $C_{m_{k}, k}$ (Fig. 1(a)), $\mathbf{v}_{t k}=\mathbf{J}_{t k} \mathbf{v}\left(\mathbf{J}_{t k}\right.$ is a matrix of dimension $\left(\left(n\left(m_{i}-1\right)\right) \times n\right)$ and this relation can be obtained by considering the rigid body displacement of any point of the robot platform),

3) combine these two relations with (5) in order to obtain

$$
\mathbf{J}_{k} \dot{\mathbf{q}}_{t}=\mathbf{J}_{t k} \mathbf{J}_{p} \dot{\mathbf{q}} \Rightarrow \dot{\mathbf{q}}_{t}=\mathbf{J}_{t} \dot{\mathbf{q}}, \mathbf{J}_{t}=\mathbf{J}_{k}^{-1} \mathbf{J}_{t k} \mathbf{J}_{p}
$$

All the previous expressions are valuable as long as the robot does not meet any singularity and as long as there are the same number of actuators as the number of platform dof to control. The singularity avoidance or crossing is not the main topic of this paper, and the reader should refer to [13], [14] for further developments. In the following of the section II-C, it is considered that all these matrices are regular.

To take into account the loop-closure constraints into the dynamic model of the parallel robot, the Lagrange multipliers $\lambda$ can be used [10] to compute the $(n \times 1)$ vector of the actuated joint force/torque $\tau_{i d m}$ of the closed-loop structure. $\tau_{i d m}$ can be obtained in relation of the Lagrange multipliers $\lambda$ by

$$
\tau_{i d m}=\left[\mathbf{0},-\mathbf{B}_{p}^{T}\right] \lambda,
$$

where $\lambda$ is calculated from the relation:

$$
\left[\begin{array}{cc}
\mathbf{J}_{k}^{T} & \mathbf{0} \\
-\mathbf{J}_{t k}^{T} & \mathbf{A}_{p}^{T}
\end{array}\right] \lambda=\mathbf{A}^{T} \lambda=\left[\begin{array}{c}
\tau_{i d m_{t}} \\
\tau_{p r}
\end{array}\right]
$$

with $\mathbf{A}$ a square matrix of dimension $\left(\left(n m_{i}\right) \times\left(n m_{i}\right)\right)$ and

$$
\tau_{p r}=\mathbf{D}^{T} \tau_{p}
$$

The relation (13), that uses the matrix $\mathbf{D}$ defined in (9), can be easily proved using the principle of virtual powers.

Solving (11) and using the right part of (4), (5) and (10), it can be demonstrated that:

$$
\begin{aligned}
\tau_{i d m} & =\mathbf{J}_{t}^{T} \tau_{i d m_{t}}+\mathbf{J}_{p}^{T} \mathbf{D}^{T} \tau_{p} \\
& =\mathbf{J}_{t}^{T} \mathbf{I D M}_{s t_{t}} \chi_{s t_{t}}+\mathbf{J}_{p}^{T} \mathbf{D}^{T} \mathbf{I D M}_{p} \chi_{p} \\
& =\left[\begin{array}{ll}
\mathbf{J}_{t}^{T} \mathbf{I D M}_{s t_{t}} & \mathbf{J}_{p}^{T} \mathbf{D}^{T} \mathbf{I D M}_{p}
\end{array}\right]\left[\begin{array}{ll}
\chi_{s t_{t}}^{T} & \chi_{p}^{T}
\end{array}\right]^{T} \\
& =\mathbf{I D M}_{s t}(\mathbf{q}, \dot{\mathbf{q}}, \ddot{\mathbf{q}}) \chi_{s t}
\end{aligned}
$$

(14) represents the inverse dynamic model of the parallel robot without actuation redundancy.

\section{INVERSE DYNAMIC IDENTIFICATION MODEL OF} PARALLEL RoBOTS WITH ACTUATION REDUNDANCY

\section{A. Computation of the IDIM}

Let us consider in this part an actuation redundant parallel robot with $n$ actuators and $r$ independent controlled $d o f$, where $r<n$. Differentiating (3) with respect to time, it can now be proved that the matrix $\mathbf{A}_{p}$ of (5) becomes rectangular of dimension $(n \times r)$, while the matrix $\mathbf{B}_{p}$ stays square of dimension $(n \times n)[1]$.

As a result, the right parts of (5) and (7) must be changed as:

$$
\mathbf{A}_{p} \mathbf{v}+\mathbf{B}_{p} \dot{\mathbf{q}}=\mathbf{0} \Rightarrow \dot{\mathbf{q}}=-\mathbf{B}_{p}^{-1} \mathbf{A}_{p} \mathbf{v}=\mathbf{J}_{p}^{i n v} \mathbf{v}
$$

or also

$$
\mathbf{v}=\mathbf{J}_{p}^{i n v}+\dot{\mathbf{q}}, \mathbf{J}_{p}^{i n v}+=\left(\mathbf{J}_{p}^{i n v}{ }^{T} \mathbf{J}_{p}^{i n v}\right)^{-1} \mathbf{J}_{p}^{i n v T}
$$

and

$$
\begin{aligned}
& \mathbf{A}_{p} \dot{\mathbf{v}}+\dot{\mathbf{A}}_{p} \mathbf{v}+\mathbf{B}_{p} \ddot{\mathbf{q}}+\dot{\mathbf{B}}_{p} \dot{\mathbf{q}}=\mathbf{0} \\
& \Rightarrow \ddot{\mathbf{q}}=-\mathbf{B}_{p}^{-1}\left(\dot{\mathbf{A}}_{p} \mathbf{v}+\mathbf{A}_{p} \dot{\mathbf{v}}+\dot{\mathbf{B}}_{p} \dot{\mathbf{q}}\right)
\end{aligned}
$$

or also

$$
\dot{\mathbf{v}}=-\mathbf{A}_{p}^{+}\left(\dot{\mathbf{A}}_{p} \mathbf{v}+\mathbf{B}_{p} \ddot{\mathbf{q}}+\dot{\mathbf{B}}_{p} \dot{\mathbf{q}}\right), \mathbf{A}_{p}^{+}=\left(\mathbf{A}_{p}^{T} \mathbf{A}_{p}\right)^{-1} \mathbf{A}_{p}^{T}
$$

Moreover, the matrix $\mathbf{A}^{T}$ of (12) becomes now a rectangular matrix with $\left(\left(n \times m_{i}-1\right)+r\right)$ rows and $\left(n \times m_{i}\right)$ columns, i.e. the system (11) has more unknowns than equations. Thus, there are infinity of solutions for the Lagrange multipliers $\lambda$. One solution can be obtained, assuming that the value of the overconstraint $\mathbf{c}$ in the robot is known [5]:

$$
\begin{aligned}
& \tau_{i d m}=\left[\mathbf{0},-\mathbf{B}_{p}^{T}\right] \lambda, \\
& \lambda=\left(\mathbf{A}^{T}\right)^{+}\left[\begin{array}{c}
\tau_{i d m_{t}} \\
\tau_{p r}
\end{array}\right]+\left(\mathbf{I}-\left(\mathbf{A}^{T}\right)^{+} \mathbf{A}^{T}\right) \mathbf{c}
\end{aligned}
$$

where $\left(\mathbf{A}^{T}\right)^{+}$is the Moore-Penrose pseudo-inverse of $\mathbf{A}^{T}$ :

$$
\left(\mathbf{A}^{T}\right)^{+}=\mathbf{A}\left(\mathbf{A}^{T} \mathbf{A}\right)^{-1}
$$

However, in the identification process, the nominal values of the inertial parameters are generally unknown and, as a result, the value of the overcontraint $\mathbf{c}$ cannot be set $a$ priori. Thus, the dynamic model (19) cannot be used as it. Nevertheless, as matrices $\mathbf{B}_{p}$ and $\mathbf{J}_{k}$ of (5) and (10) are still square, (19) can be rewritten as:

$$
\left[\begin{array}{c}
\tau_{i d m} \\
\tau_{i d m_{t}}
\end{array}\right]=\left[\begin{array}{cc}
\mathbf{0} & -\mathbf{B}_{p}^{T} \\
\mathbf{J}_{k}^{T} & \mathbf{0}
\end{array}\right] \lambda, \quad\left[\begin{array}{ll}
-\mathbf{J}_{t k}^{T} & \mathbf{A}_{p}^{T}
\end{array}\right] \lambda=\tau_{p r}
$$

or also

$$
\tau_{p r}=\left[\begin{array}{ll}
-\mathbf{J}_{t k}^{T} & \mathbf{A}_{p}^{T}
\end{array}\right]\left[\begin{array}{cc}
\mathbf{0} & \mathbf{J}_{k}^{-T} \\
-\mathbf{B}_{p}^{-T} & \mathbf{0}
\end{array}\right]\left[\begin{array}{c}
\tau_{i d m} \\
\tau_{i d m_{t}}
\end{array}\right]
$$

Developing (22), it comes that:

$$
\begin{aligned}
\tau_{p r} & =\left[\begin{array}{ll}
-\mathbf{A}_{p}^{T} \mathbf{B}_{p}^{-T} & -\mathbf{J}_{t k}^{T} \mathbf{J}_{k}^{-T}
\end{array}\right]\left[\begin{array}{c}
\tau_{i d m} \\
\tau_{i d m_{t}}
\end{array}\right] \\
& =\left[\begin{array}{lll}
\mathbf{J}_{p}^{i n v} & T & -\mathbf{J}_{t k}^{T} \mathbf{J}_{k}^{-T}
\end{array}\right]\left[\begin{array}{c}
\tau_{i d m} \\
\tau_{i d m_{t}}
\end{array}\right]
\end{aligned}
$$


Introducing (1), (2) and (13) into (23) leads to:

$$
\begin{aligned}
\mathbf{J}_{p}^{i n v T^{T} \tau_{i d m}} & =\mathbf{J}_{t k}^{T} \mathbf{J}_{k}^{-T} \tau_{i d m_{t}}+\mathbf{D}^{T} \tau_{p} \\
& =\mathbf{J}_{t k}^{T} \mathbf{J}_{k}^{-T} \mathbf{I D M}_{s t_{t}} \chi_{s t_{t}}+\mathbf{D}^{T} \mathbf{I D M}_{p} \chi_{p} \\
& =\left[\begin{array}{ll}
\mathbf{J}_{t k}^{T} \mathbf{J}_{k}^{-T} \mathbf{I D M}_{s t_{t}} & \mathbf{D}^{T} \mathbf{I D M}_{p}
\end{array}\right]\left[\begin{array}{ll}
\chi_{s t_{t}}^{T} & \chi_{p}^{T}
\end{array}\right]^{T} \\
& =\mathbf{I D M}_{s t}^{\text {red }}(\mathbf{q}, \dot{\mathbf{q}}, \ddot{\mathbf{q}}) \chi_{s t}
\end{aligned}
$$

Thus, by projecting the input forces/torques on the platform coordinates through the use of the matrix $\mathbf{J}_{p}^{i n v} T$, the inverse dynamic model becomes unique and can thus be used for identification purpose.

The model (24) can be simplified through the use of the identifiable parameters. The identifiable parameters are the base parameters which are the minimum number of dynamic parameters from which the dynamic model can be calculated [10]. The minimal dynamic model can be written using the $n_{b}$ base dynamic parameters $\chi$ as follows:

$$
\mathbf{J}_{p}^{i n v}{ }^{T} \tau_{i d m}=\operatorname{IDM}(\mathbf{q}, \dot{\mathbf{q}}, \ddot{\mathbf{q}}) \chi
$$

where IDM is a subset of independent columns in $\mathbf{I D M} \mathbf{M}_{s t}^{\text {red }}$ which defines the identifiable parameters.

Finally, because of perturbations due to noise measurement and modeling errors, the actual force/torque $\tau$ differs from $\tau_{i d m}$ by an error e, such that:

$$
\mathbf{J}_{p}^{i n v T^{T}} \tau=\mathbf{J}_{p}^{i n v}{ }^{T} \tau_{i d m}+\mathbf{e}=\mathbf{I D M} \chi+\mathbf{e}
$$

where $\tau$ is calculated with the drive chain relation:

$$
\tau=\mathbf{v}_{\tau} \mathbf{g}_{\tau}=\operatorname{diag}\left(v_{\tau}^{j}\right)\left[\begin{array}{lll}
g_{\tau}^{1} & \cdots & g_{\tau}^{n}
\end{array}\right]^{T}
$$

$\mathbf{v}_{\tau}$ is the $(n \times n)$ matrix of the actual motor current references of the current amplifiers $\left(v_{\tau}^{j}\right.$ corresponds to actuator $j)$ and $\mathbf{g}_{\tau}$ is the $(n \times 1)$ vector of the joint drive gains $\left(g_{\tau}^{j}\right.$ corresponds to actuator $j$ ) that is given by a priori manufacturer's data or using some special procedures [15], [16]. Equation (26) represents the Inverse Dynamic Identification Model (IDIM).

\section{B. IDIM Including the Payload}

The payload is considered as an additional link (denoted as link $l$ ) fixed to the robot platform [9]. The model (25) becomes:

$$
\mathbf{J}_{p}^{i n v} T_{\tau} \tau=\left[\begin{array}{ll}
\mathbf{I D M} & \mathbf{I D M}_{l}
\end{array}\right]\left[\begin{array}{c}
\chi \\
\chi_{l}
\end{array}\right]+\mathbf{e}=\mathbf{I D M}_{t o t} \chi_{t o t}+\mathbf{e}
$$

where:

- $\chi_{l}$ is the $(10 \times 1)$ vector of the inertial parameters of the payload;

- $\mathbf{I D M}_{l}$ is the $(r \times 10)$ Jacobian matrix of $\mathbf{J}_{p}^{i n v} T_{i d m}$, with respect to the vector $\chi_{l}$.

\section{Usual IDENTIFICATION PROCEDURE}

This part presents some necessary recalls on the identification procedure.

\section{A. Recalls on Least Squares Identification of the Dynamic Parameters (IDIM-LS)}

The off-line identification of the base dynamic parameters $\chi$ is considered, given measured or estimated off-line data for $\tau$ and $(\mathbf{q}, \dot{\mathbf{q}}, \ddot{\mathbf{q}})$, collected while the robot is tracking some planned trajectories. The model (26) is sampled at frequency $f_{m}$ in order to get an over-determined linear system of $r_{f_{m}}$ equations and $n_{b}$ unknowns:

$$
\mathbf{Y}_{f_{m}}(\tau)=\mathbf{W}_{f_{m}}(\hat{\mathbf{q}}, \hat{\dot{\mathbf{q}}}, \hat{\ddot{\mathbf{q}}}) \chi+\rho_{f_{m}}
$$

where $(\hat{\mathbf{q}}, \hat{\dot{\mathbf{q}}}, \hat{\ddot{\mathbf{q}}})$ is an estimation of $(\mathbf{q}, \dot{\mathbf{q}}, \ddot{\mathbf{q}})$, respectively, obtained by sampling and band-pass filtering the measure of $\mathbf{q}$ [17], $\rho_{f_{m}}$ is the $\left(r_{f_{m}} \times 1\right)$ vector of errors, $\mathbf{Y}_{f_{m}}$ is the $\left(r_{f_{m}} \times 1\right)$ vector of the inputs (computed using the relation $\mathbf{J}_{p}^{i n v} T^{T} \tau$ from (26) and (27)) ${ }^{1}$, sampled at frequency $f_{m}$ and $\mathbf{W}_{f_{m}}(\hat{\mathbf{q}}, \hat{\dot{\mathbf{q}}}, \hat{\mathbf{q}})$ is the $\left(r_{f_{m}} \times n_{b}\right)$ observation matrix.

The force/torque $\tau$ is perturbed by high frequency unmodelled friction and flexibility force/torque of the joint drive chain which is rejected by the closed loop control. These force/torque ripples are eliminated with a parallel decimation procedure which low pass filters in parallel $\mathbf{Y}_{f_{m}}$ and each column of $\mathbf{W}_{f_{m}}$ and resamples them at a lower rate, keeping one sample over $n_{d}$. This parallel decimation can be carried out with the MATLAB decimate function, where the low pass filter cutoff frequency, $\omega_{f p}=2 \pi 0.8 f_{m} /\left(2 n_{d}\right)$, is chosen in order to keep $\mathbf{Y}_{f_{m}}$ and $\mathbf{W}_{f_{m}}$ in the same frequency range of the model dynamics. After the data acquisition procedure and the parallel decimation of (29), we obtain the overdetermined linear system

$$
\mathbf{Y}(\tau)=\mathbf{W}(\hat{\mathbf{q}}, \hat{\dot{\mathbf{q}}}, \hat{\ddot{\mathbf{q}}}) \chi+\rho
$$

where $\rho$ is the $\left(r_{c} \times 1\right)$ vector of errors, $\mathbf{Y}$ is the $\left(r_{c} \times 1\right)$ vector of the input torques/force and $\mathbf{W}(\hat{\mathbf{q}}, \hat{\dot{\mathbf{q}}}, \hat{\ddot{\mathbf{q}}})$ is the $\left(r_{c} \times n_{b}\right)$ observation matrix.

It is to be noted that no error is introduced by the parallel filtering process in the linear relation (30) compared with (29). In [17], practical rules for tuning this filter are given.

Using the base parameters and tracking 'exciting' reference trajectories, i.e. optimized trajectories that can be computed by nonlinear minimization of a criterion function of the condition number of the $\mathbf{W}$ matrix [18], a wellconditioned matrix $\mathbf{W}$ can be obtained. The $L S$ solution $\hat{\chi}$ of (30) is given by:

$$
\hat{\chi}=\mathbf{W}^{+} \mathbf{Y}, \text { where } \mathbf{W}^{+}=\left(\mathbf{W}^{T} \mathbf{W}\right)^{-1} \mathbf{W}^{T}
$$

It is computed using the $Q R$ factorization of $\mathbf{W}$.

Standard deviations $\sigma_{\hat{\chi}_{i}}$ can be estimated assuming that $\mathbf{W}$ is a deterministic matrix and $\rho$ is a zero mean additive independent noise [17], with a covariance matrix $\mathbf{C}_{\rho \rho}$ such that

$$
\mathbf{C}_{\rho \rho}=E\left(\rho \rho^{T}\right)=\sigma_{\rho}^{2} \mathbf{I}_{r}
$$

${ }^{1}$ The use of the relation $\mathbf{J}_{p}^{i n v} T_{\tau}$ for sampling the vector $\mathbf{Y}$ instead of the values of the input forces/torques $\tau$ is the main difference with the usual identification procedure for serial and parallel robots without actuation redundancy. 
$E$ is the expectation operator and $\mathbf{I}_{r}$, the $r_{c} \times r_{c}$ identity matrix. An unbiased estimation of the standard deviation $\sigma_{\rho}$ is:

$$
\sigma_{\rho}^{2}=\|\mathbf{Y}-\mathbf{W} \hat{\chi}\|^{2} /\left(r_{c}-n_{b}\right)
$$

The covariance matrix of the estimation error is given by:

$$
\mathbf{C}_{\hat{\chi} \hat{\chi}}=E\left[(\chi-\hat{\chi})(\chi-\hat{\chi})^{T}\right]=\sigma_{\rho}^{2}\left(\mathbf{W}^{T} \mathbf{W}\right)^{-1}
$$

$\sigma_{\hat{\chi}_{i}}^{2}=\mathbf{C}_{\hat{\chi} \hat{\chi}}(i, i)$ is the $i$-th diagonal coefficient of $\mathbf{C}_{\hat{\chi} \hat{\chi}}$ (34).

The ordinary $L S$ can be improved by taking into account different standard deviations on actuated joint $j$ equations errors [17]. Data in $\mathbf{Y}$ and $\mathbf{W}$ of (30) are weighted with the inverse of the standard deviation of the error calculated from ordinary $L S$ solution of the equations of joint $j$ [17]

$$
\mathbf{Y}^{j}=\mathbf{W}^{j} \chi+\rho^{j}
$$

This weighting operation normalizes the errors in (30) and gives the weighted $L S$ estimation of the parameters (IDIM$W L S)$.

\section{B. Payload Identification}

In order to identify both the robot and the payload dynamic parameters, using the model (28), it is necessary that the robot carried out two types of trajectories [19]:

1) trajectories without the payload, and

2) trajectories with the payload fixed to the end-effector.

The sampling and filtering of the model IDIM (28) can be then written as:

$$
\mathbf{Y}=\left[\begin{array}{cc}
\mathbf{W}_{a} & \mathbf{0} \\
\mathbf{W}_{b} & \mathbf{W}_{l}
\end{array}\right]\left[\begin{array}{c}
\chi \\
\chi_{l}
\end{array}\right]+\rho
$$

where

- $\mathbf{W}_{a}$ is the observation matrix of the robot in the unloaded case,

- $\mathbf{W}_{b}$ is the observation matrix of the robot in the loaded case,

- $\mathbf{W}_{l}$ is the observation matrix of the robot corresponding to the payload inertial parameters.

Thus, these two types of trajectories avoid the regrouping of the payload parameters with those of the platform and allow their independent identification. Next section presents experimental results on a prototype of actuation redundant parallel robot.

\section{CASe Study}

\section{A. Description of the DualV}

The DualV (Fig. 2) is a prototype of planar parallel robot with actuation redundancy developed at the LIRMM [11]. This robot has 3 controlled dof (two translations in the plane $(x O y)$ and one rotation around the $z$ axis) but 4 legs, with one actuator by leg. Thus, its degree of redundancy is equal to 1. Each leg is composed of one proximal and one distal link. The proximal link is attached to the base by one actuated revolute joint and to the distal link by one passive revolute joint. The distal link is also attached to the moving platform by one passive revolute joint.

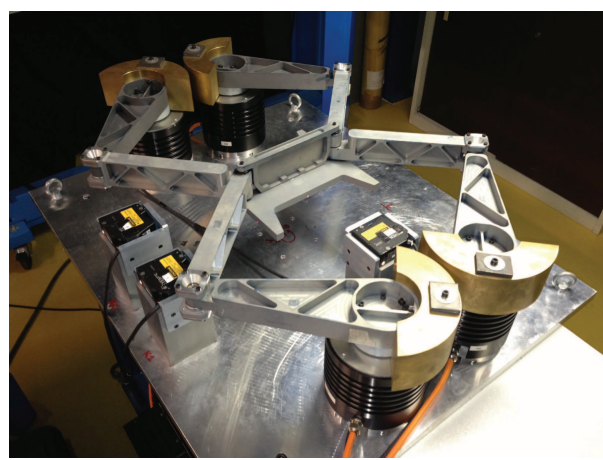

(a) The prototype

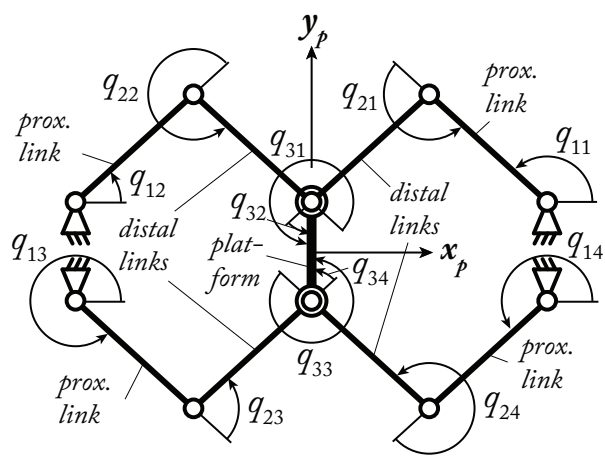

(b) Kinematic description

Fig. 2. The DualV.

The geometric parameters of the virtual open-loop tree structure are described in Table I using the modified Denavit and Hartenberg notation $(M D H)$ [10] (in this table, $\gamma_{1}=15.52 \mathrm{deg}, \gamma_{2}=164.48 \mathrm{deg}, \gamma_{3}=-164.48 \mathrm{deg}$ and $\gamma_{4}=-15.52 \mathrm{deg}$ ). The platform and payload are considered as supplementary bodies, the payload being fixed on the platform. They are respectively numbered as bodies 4 and 5.

The $M D H$ notation being well known, the parameters of Table I will not be defined here. For more information concerning the $M D H$ parameters, the reader should refer to [10].

TABLE I

MDH PARAMETERS FOR THE FRAMES CORRESPONDING TO $i$-TH ROBOT

$$
\text { LEG }(i=1, \ldots, 4) \text {. }
$$

\begin{tabular}{rrrrrrrr}
\hline \hline$j_{i}$ & $a_{j_{i}}$ & $\mu_{j i}$ & $\sigma_{j i}$ & $\gamma_{j i}$ & $d_{j i}$ & $\theta_{j i}$ & $r_{j i}$ \\
\hline $1_{i}$ & 0 & 1 & 0 & $\gamma_{i}$ & $d_{1}=0.41 \mathrm{~m}$ & $q_{1 i}-\gamma_{i}$ & 0 \\
$2_{i}$ & $1_{i}$ & 0 & 0 & 0 & $d_{2}=0.28 \mathrm{~m}$ & $q_{2 i}$ & 0 \\
$3_{i}$ & $2_{i}$ & 0 & 0 & 0 & $d_{3}=0.28 \mathrm{~m}$ & $q_{3 i}$ & 0 \\
\hline \hline
\end{tabular}

The DualV is actuated by four ETEL RTMB0140-100 direct drive actuators, which can deliver maximal torques of $127 \mathrm{Nm}$. The robot is able to achieve accelerations of $25 \mathrm{~g}$ in its workspace. The current amplifier can provide directly the measure of the input torque produced by the actuator. The controller of the DualV was developed within the framework of an industrial $\mathrm{PhD}$ thesis which is still confidential for now [20], therefore we are not able to give further explanations 

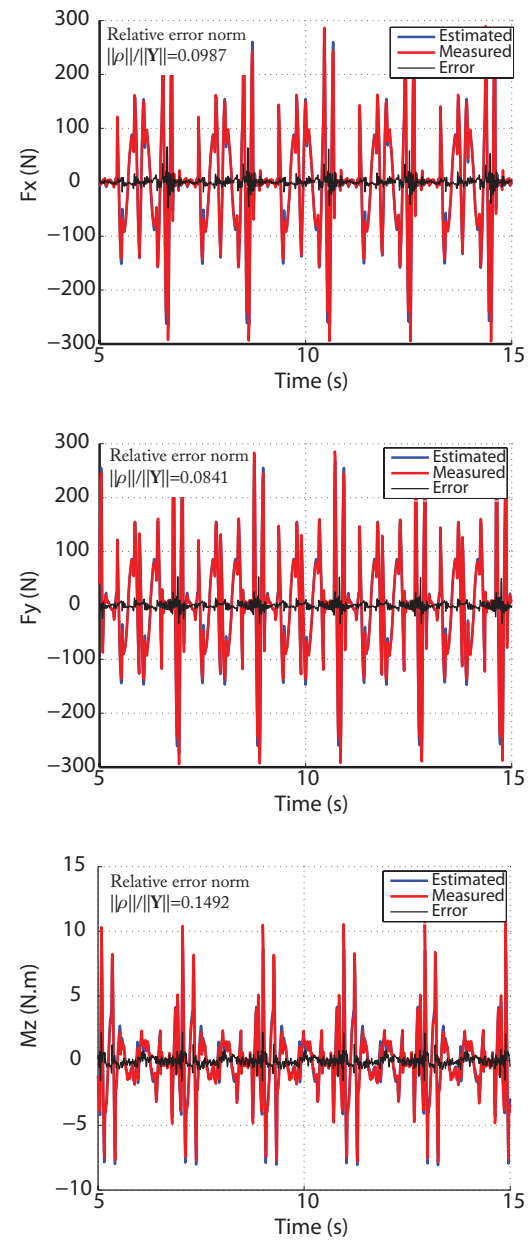

Fig. 3. Projections of the input torques on the platform, estimated from input torques using (26) (red lines) and rebuilt using identified parameters (blue lines) with the payload of $5.37 \mathrm{~kg}$.

on this point.

\section{B. Identification Results}

In this part, experimentations are performed and the dynamic model identification is carried out on the DualV using the modeling approach presented in Section III and the identification procedure proposed in Section IV. To estimate the quality of the identification procedure, a payload mass of $5.37 \mathrm{~kg}$ which has been accurately weighed is mounted of the platform and will be identified in the same time as the robot parameters.

Even paying attention to the choice of the exciting trajectories, some small parameters remain poorly identifiable because they have no significant contribution in the joint torques. These parameters have no significant estimations and can be canceled in order to simplify the dynamic model. Thus parameters such that the relative standard deviation $\% \sigma_{\hat{\chi}_{r i}}$ is too high are canceled to keep a set of essential parameters of a simplified dynamic model with a good accuracy [19]. The essential parameters are calculated using an iterative procedure starting from the base parameters estimation. At each step the base parameter which has the
TABLE II

ESSENTIAL PARAMETERS OF THE DUALV.

\begin{tabular}{rrrr}
\hline \hline Param. & A priori & Id. Val. & $\% \sigma_{\hat{\chi}_{r i}}$ \\
\hline$z z_{11 R}$ & $4.92 e-2$ & $4.45 e-2$ & 1.58 \\
$z z_{12 R}$ & $4.92 e-2$ & $4.81 e-2$ & 1.39 \\
$z z_{13 R}$ & $4.92 e-2$ & $4.81 e-2$ & 1.41 \\
$z z_{14 R}$ & $4.92 e-2$ & $5.07 e-2$ & 1.38 \\
$z z_{4}$ & $2.16 e-2$ & $2.06 e-2$ & 1.37 \\
$m_{4}$ & $2.00 e+0$ & $1.92 e+0$ & 1.08 \\
$z z_{5}$ & N/A & $1.61 e-2$ & 1.71 \\
$m x_{5}$ & N/A & $-1.27 e-1$ & 0.76 \\
$m_{5}$ & $5.37 e+0$ & $5.42 e+0$ & 0.30 \\
\hline \hline
\end{tabular}

largest relative standard deviation is canceled. A new IDIM$W L S$ parameter estimation of the simplified model is carried out with new relative error standard deviations $\% \sigma_{\hat{\chi}_{r i}}$. The procedure ends when $\max \left(\% \sigma_{\hat{\chi}_{r i}}\right) / \min \left(\% \sigma_{\hat{\chi}_{r i}}\right)<r_{\sigma}$, where $r_{\sigma}$ is a ratio ideally chosen between 10 and 30 depending on the level of perturbation in $\mathbf{Y}$ and $\mathbf{W}$. In the following of the paper, this ratio is fixed to 10 .

Table II presents the identification results. Subscript ' $R$ ' stands for the parameters that have been regrouped using the procedure presented in Section III. It can be observed that the robot parameters have been correctly estimated and that the identified values are very close from the a priori values. Moreover, the payload of $5.37 \mathrm{~kg}$ has been very accurately identified.

The projections of the input torques on the platform are shown in Fig. 3 for a trajectory different from the one used for the identification process (i.e. the results are crossvalidated). It can be observed that they are well rebuilt. Finally, the value the measured input torques, the estimated input torques and the value of the overconstraint $\mathbf{c}$ computed using expressions (19) and (20) are shown at Figs. 4 and 5. It can be seen that the estimated torques are very close to the measured ones and that the average overconstraint $\mathbf{c}$ in the robot legs is about $10 \mathrm{~N}$.

\section{CONCLUSION}

This paper has presented a method for the identification of the inertial parameters of parallel robots with actuation redundancy. Contrary to serial robots or parallel robots without actuation redundancy for which the dynamic identification methods are based on the use of the IDIM which calculates the joint forces/torques that are linear in relation to the dynamic parameters, for actuation redundant parallel robot that are over-constrained, the IDIM has infinity of solutions for the force/torque prediction, depending of the value of the desired overconstraint that is a priori unknown. As a result, the IDIM cannot be used as it.

This paper proposed a modified formulation for the IDIM of actuation redundant robots that can be used for identification purpose. This formulation consists of projecting the input torques/forces on the platform coordinates, thus leading to a unique solution of the model that can thus be used in the identification process. The identification of the inertial parameters of a planar parallel robot with actuation redundancy, the DualV, was then performed using this modified IDIM. 

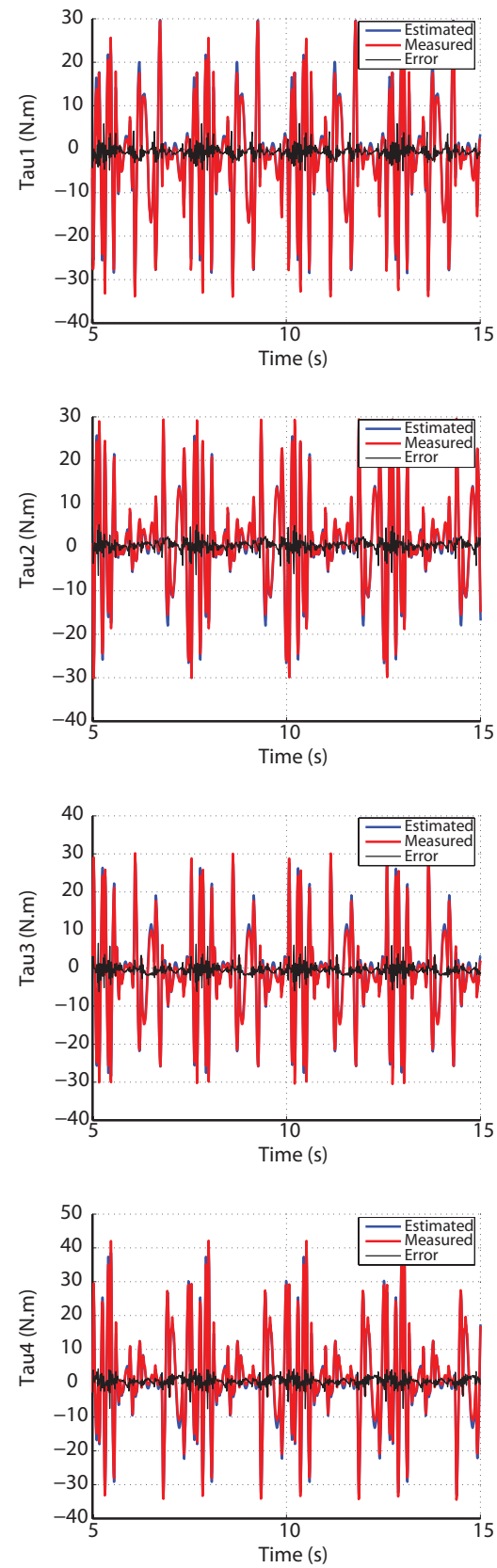

Fig. 4. Measured (red lines) and estimated (blue lines) input torques rebuilt using identified parameters with the payload of $5.37 \mathrm{~kg}$.

Experimental results show that the inertial parameters of the robot were correctly identified. Moreover, for validation purpose, a known payload mass has been added on the robot to be sure that the identification process was correct. This mass has been very accurately identified. Finally, it has been shown that the torque prediction with the newly identified parameters was correct.

\section{REFERENCES}

[1] J. Merlet, Parallel Robots. Springer, 2nd ed., 2006.

[2] B. Yi, R. Freeman, and D. Tesar, "Force and stiffness transmision in redundantly actuated mechanisms: The case for a spherical shoulder mechanism," Robotics, Spatial Mechanisms, Mechanical Systems, vol. 45, pp. 163-172, 1994.

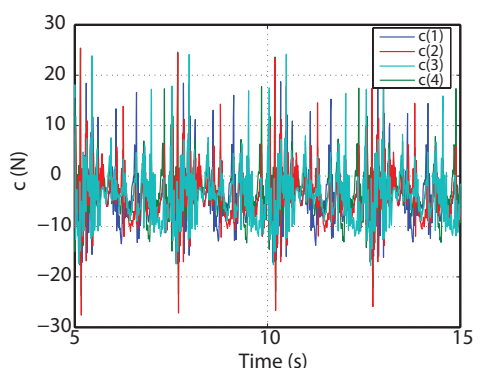

Fig. 5. Value of the overconstraint.

[3] R. Kurtz and V. Hayward, "Multiple-goal kinematic optimization of a parallel spherical mechanism with actuator redundancy," IEEE Transactions on Robotics and Automation, vol. 8, no. 5, pp. 644-651, 1992.

[4] M. A. Nahon and J. Angeles, "Force optimization in redundantlyactuated closed kinematic chains," in Proceedings of the International Conference on Robotics and Automation, 1989.

[5] A. Muller, "Internal preload control of redundantly actuated parallel manipulators - its application to backlash avoiding control," IEEE Transactions on Robotics, vol. 21, no. 4, pp. 668-677, 2005.

[6] H. Cheng, Y.-K. Yiu, and Z. Li, "Dynamics and control of redundantly actuated parallel manipulators," IEEE/ASME Trans. on Mechatronics, vol. 8, no. 4, pp. 483-491, 2003.

[7] M. A. Hanon, "Comparison of methods for the control of redundantlyactuated robotic systems," Journal of Intelligent and Robotic Systems, vol. 14 , pp. 3-21, 1995.

[8] A. Muller, "Internal prestress control of redundantly actuated parallel manipulators its application to backlash avoiding control," IEEE Transactions on Robotics, vol. 21, no. 4, pp. 668-677, 2005.

[9] W. Khalil, M. Gautier, and P. Lemoine, "Identification of the payload inertial parameters of industrial manipulators," in Proceedings IEEE ICRA, (Roma, Italy), pp. 4943-4948, April 2007.

[10] W. Khalil and E. Dombre, Modeling, Identification and Control of Robots. Hermes Penton London, 2002.

[11] V. van der Wijk, S. Krut, F. Pierrot, and J. Herder, "Generic method for deriving the general shaking force balance conditions of parallel manipulators with application to a redundant planar 4-rrr parallel manipulator," in Proceedings of the 13th World Congress in Mechanism and Machine Science, (Guanajuato, Mexico), June 19-25 2011.

[12] S. Briot and M. Gautier, "Global identification of drive gains and dynamic parameters of parallel robots - part 1: Theory," in Proceedings of the 19th CISM-IFToMM Symposium on Robot Design, Dynamics, and Control (RoManSy), 2012.

[13] S. Briot and V. Arakelian, "Optimal force generation of parallel manipulators for passing through the singular positions," International Journal of Robotics Research, vol. 27, no. 8, pp. 967-983, 2008.

[14] D. N. Nenchev, S. Bhattacharya, and M. Uchiyama, "Dynamic analysis of parallel manipulators under the singularity-consistent parameterization," Robotica, vol. 15, no. 4, pp. 375-384, 1997.

[15] P. Corke, "In situ measurement of robot motor electrical constants," Robotica, vol. 23, no. 14, pp. 433-436, 1996.

[16] M. Gautier and S. Briot, "New method for global identification of the joint drive gains of robots using a known payload mass," in Proceedings IEEE IROS, (San Francisco, CA, USA), September 2011.

[17] M. Gautier, "Dynamic identification of robots with power model," in Proceedings IEEE ICRA, (Albuquerque, USA), pp. 1922-1927, April 1997.

[18] M. Gautier and W. Khalil, "Exciting trajectories for the identification of the inertial parameters of robots," International Journal of Robotics Research, vol. 11, no. 4, pp. 362-375, 1992.

[19] W. Khalil and O. Ibrahim, "General solution for the dynamic modeling of parallel robots," Journal of Intelligent and Robotic Systems, vol. 49, no. 1, pp. 19-37, 2007.

[20] H. Yang, Agile mobile manufacturing for large workpieces (Etude dun systeme de fabrication agile mobile pour composants de grande taille). $\mathrm{PhD}$ thesis, Montpellier Sciences and Technics University (UM2), Montpellier, France, May 10th 2012. 